\title{
Ocean One: A Robotic Avatar for Oceanic Discovery
}

\author{
Oussama Khatib, Xiyang Yeh, Gerald Brantner, Brian Soe, Boyeon Kim and Shameek Ganguly ${ }^{1}$ \\ Hannah Stuart, Shiquan Wang and Mark Cutkosky ${ }^{2}$ \\ Aaron Edsinger, Phillip Mullins and Mitchell Barham ${ }^{3}$ \\ Christian Voolstra and Khaled Salama ${ }^{4}$ \\ Michel L'Hour ${ }^{5}$ and Vincent Creuze ${ }^{6}$
}

\section{INTRODUCTION}

The promise of oceanic discovery has intrigued scientists and explorers, whether to study underwater ecology and climate change, or to uncover natural resources and historic secrets buried deep at archaeological sites. This quest to explore the oceans requires skilled human access, yet much of it is inaccessible to human divers as nearly nine-tenths of the ocean floor is at one kilometer or deeper [1]. Accessing these depths is imperative since factors such as pollution and deep-sea trawling threaten ecology and archaeological sites. These needs demand a system that deploys human-level expertise at the depths but remotely operated vehicles (ROVs) are inadequate for the task - a robotic avatar could go where humans can not, and yet embody human intelligence and intentions through immersive interfaces.

To meet the challenge of accessing oceanic depths, Stanford University, working with KAUST's Red Sea Research Center and MEKA Robotics, developed Ocean One (Fig. 1), a bimanual force-controlled humanoid robot that affords immediate and intuitive haptic interaction in oceanic environments. Teaming with the French Ministry of Culture's Underwater Archaeology Research Department, Stanford deployed Ocean One in an expedition in the Mediterranean to Louis XIV's flagship Lune, lying at ninety-one meters depth off the coast of Toulon. Following extensive testing at Stanford University, Ocean One was flown to France in the spring of 2016 for its maiden deployment, where it became the first robot avatar to embody a human's presence at the seabed.

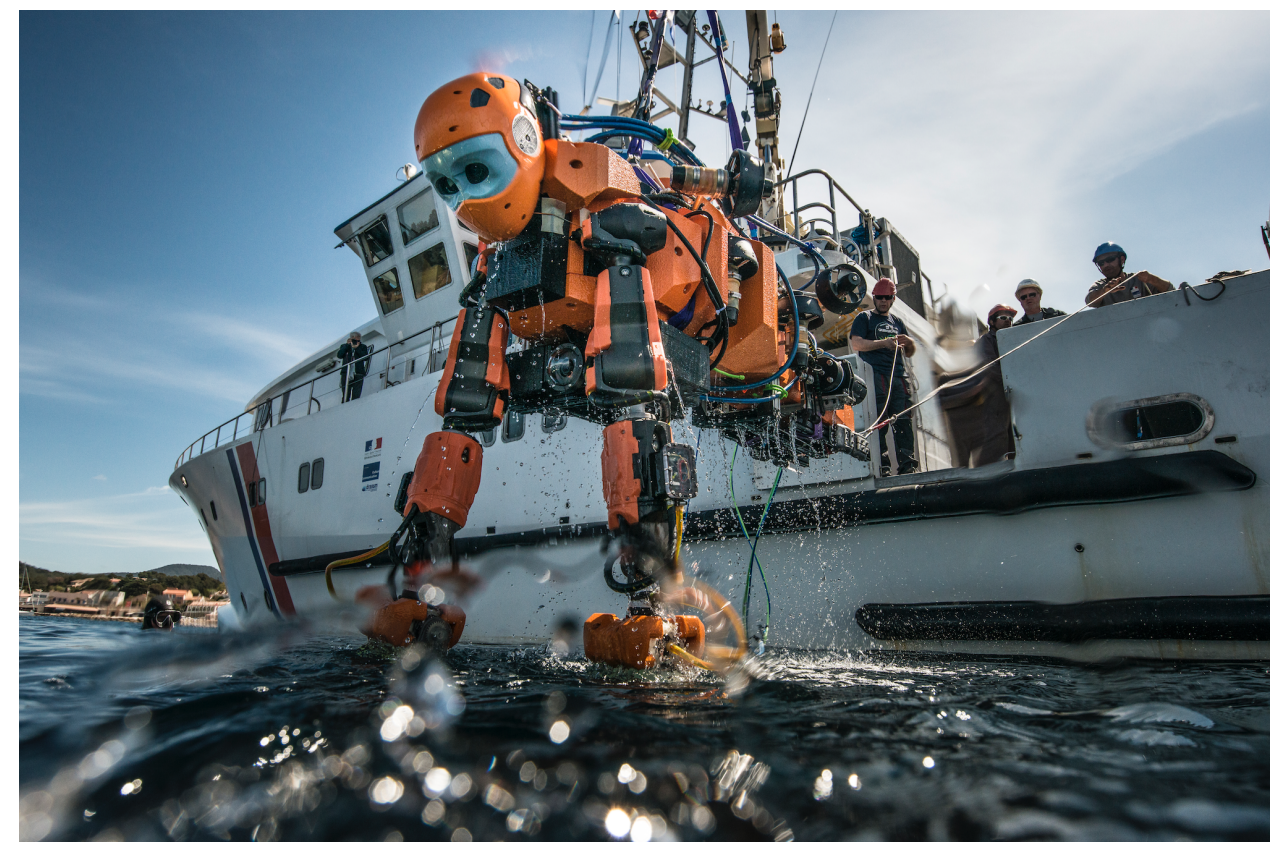

Fig. 1. Deploying Ocean One from the ship, André Malraux, in preparation for its mission to Louis XIV's flagship, Lune.

\footnotetext{
${ }^{1}$ Stanford Robotics Lab, Department of Computer Science, Stanford University, USA

2 Biomimetic and Dexterous Manipulation Lab, Department of Mechanical Engineering, Stanford University, USA

3 Meka Robotics, USA

${ }^{4}$ King Abdullah University of Science and Technology (KAUST), Saudi Arabia

${ }^{5}$ DRASSM, Ministry of Culture, France

${ }^{6}$ LIRMM, University of Montpellier, France
} 
Exploration in the oceans' depths requires human skills, expertise, and intelligence. It is, however, neither safe nor practical for divers to operate beyond fifty meters-the deeper humans venture the more complex the precautions needed to safeguard their presence and the less time they have to operate. In contrast, marine ROVs can readily access the depths, but possess limited manipulation abilities and require the constant attention of skilled pilots teleoperating their every move. The inability of ROVs to interact gently with biological specimens and fragile artifacts render them unsuitable for marine discovery and exploration. A diving machine that can embody a human, harnessing human intuition and cognition, promises to unlock the oceans' depths.

OUtside of structured environments, human reasoning remains crucial to accomplishing complex manipulation tasks. The Ocean One Avatar concept is to create a robotic diver with a high degree of autonomy in physical interaction, connected to a human expert through an intuitive interface. The robot is designed with advanced skills to interact with its environment. The human typically instructs the robot through high-level cognitive guidance but is able to intervene at any level of operation. This is made possible by building a rich interface for the human operator, one that provides visual and haptic feedback together with a user command center (UCC) that displays data from other sensors and sources (Fig. 3).

A tethered connection from the interface to the robot risks entanglement, and introduces disturbances. Rather, we envision a relay station that connects the robot to the interface through short-distance high-speed optical wireless links. In addition, the station provides for nearby recharge, and frees the robot for wider and safer operation. This concept extends to a team of untethered robots, possibly working with human divers (Fig. 2).

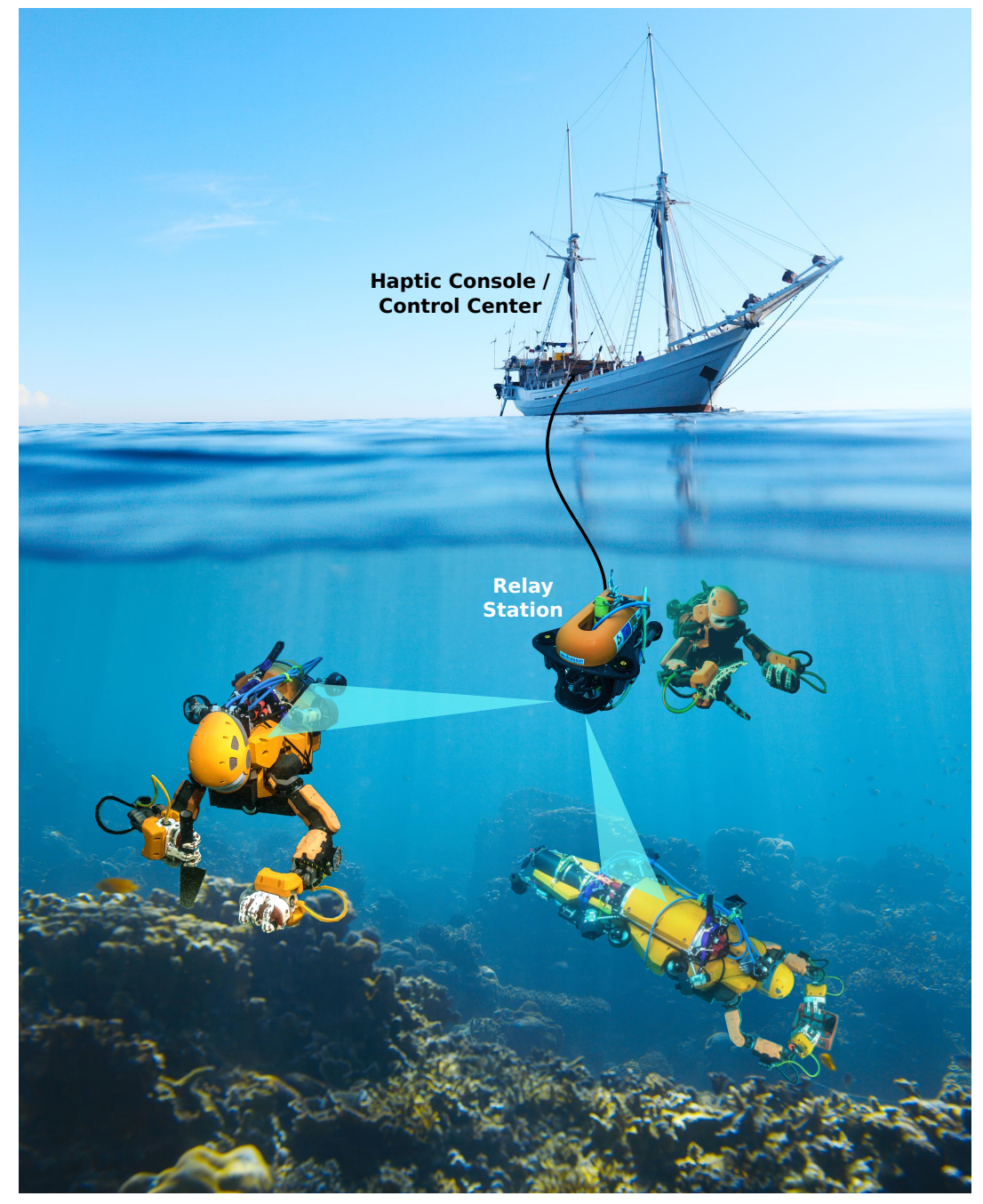

Fig. 2. A team of oceanic avatars envisioned at work with a human expert overseeing operations. The team of robots are connected through short-distance high-speed optical communication by a relay station that also provides power recharge. 


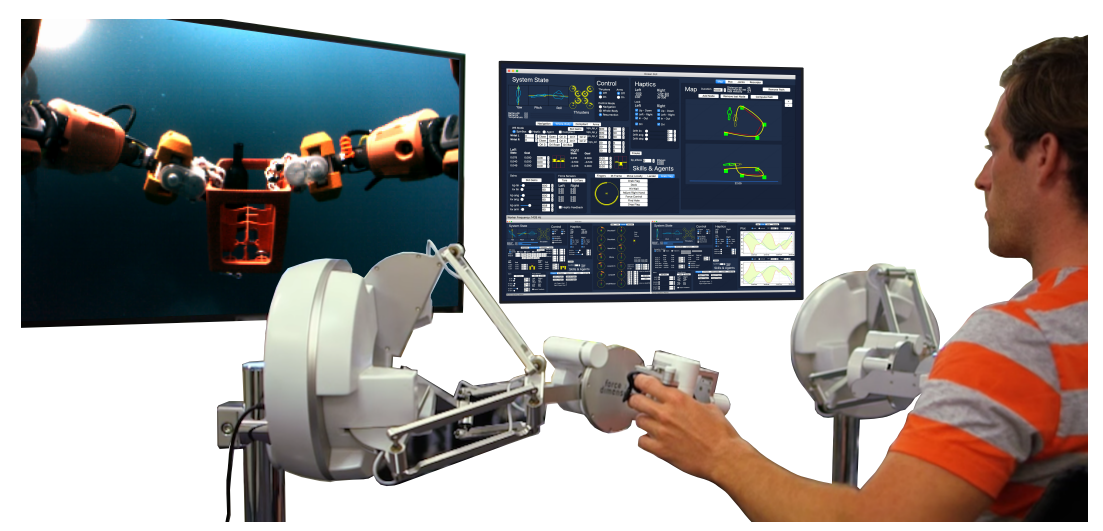

Fig. 3. A human operator guiding the robot in an underwater bimanual task through the haptic-visual interface consisting of two Sigma.7 haptic devices [8], a stereoscopic 3D display, foot pedals and the graphical user command center.

\section{HARDWARE DESIGN}

The field of ROVs has seen major advancements in navigation and sensing over the last decades. The need for expanding subsea operations has further led to the development of advanced underwater manipulators, such as Schilling's ATLAS 7R hydraulic arms [19], the SAUVIM MARIS 7080 electrical arms, and more recently, the ECA group's ARM 5E lightweight arm [10]. The SAUVIM [2] and RAUVI [18] projects have used similar arms on custom-designed underwater vehicles for autonomous intervention tasks. These tasks typically involve operating industrial equipment underwater, and employ stiff and rigid manipulators.

Ocean One, in contrast, is designed to perform marine tasks in place of human divers, with comparable capability in underwater manipulation. The robot is to accomplish operations common to human divers which include assembly of structures, and delicate handling of samples, artifacts and other irregularly-shaped objects. The design of Ocean One tackles this challenge by fully integrating navigation with bimanual manipulation and stereo-vision capabilities. The lower-body is designed for efficient underwater navigation, while the upper-body is conceived in an anthropomorphic form that offers a transparent embodiment of the human's interactions with the environment through the haptic-visual interface. Fig. 4 shows a schematic of the various system components.

Each arm of Ocean One employs seven joints with a one degree-of-freedom (DOF) underactuated hand, and the head is designed with two DOFs of pan and tilt. The body is actuated by eight thrusters, four on each side of the body. Four thrusters control the yaw motion and planar translations, while four others control the vertical translation, pitch and roll. This thruster redundancy allows full maneuverability in the event of a single thruster failure.

In an arm-body system such as Ocean One, the whole system inertial properties reflected at the hand have as upper bound those of the arm alone. This reduced effective inertia [13] can be used in a macro-mini dynamic coordination to increase task responsiveness (Fig. 6). The workspace and placement of the arms with respect to the body are optimized to maximize the range over which the faster dynamics of the arms can be used to accommodate the slower dynamics of the body. For instance, the robot is able to stabilize the task, while the body is subject to large disturbance forces such as sea currents. The macro-mini dynamic coordination also improves the ability of the robot to interact with its environment. Fig. 5 shows a simulated docking sequence where the robot comes in to grasp a hand-hold with both hands.

Ocean One uses a pair of 7-DOF compliant, electrically driven, torque-controlled arms with high torque capacities adapted from the Meka arm design. The arms are fitted with series elastic actuators (SEAs) that provide torque feedback to enhance compliant motion, force control and safety for in-contact manipulation tasks [17]. Low output impedance from the transmission also reduces impact loads on both the environment and the manipulator in the event of a collision [22]. We use low friction dynamic O-rings [16] at the output shaft of each link to minimize transmission loss.

In contrast to most underwater robots, which use stiff grippers, the hands of Ocean One are designed to be gentle enough to handle organic samples and artifacts, yet strong enough to hold tools securely, to brace at handholds, and to be impact resilient. Given these considerations, compliant underactuated hands are a natural choice and have demonstrated stability, adaptability, and impact robustness in various other applications [3], [6], [9], [12], [15], [21].

To reduce weight and complexity, the hands of Ocean One use a single motor to drive three fingers. The motor is housed in an oil-filled chamber with a single shaft seal; the fingers and tendons are wet (Fig. 7). The motor drives a shaft with three spring-loaded pulleys that wind the finger tendons. In most cases, soft transmission springs are preferred to help the fingers settle independently on irregular objects. However, when lifting heavy artifacts or extracting an object from sediment, a high transmission stiffness is useful. In Ocean One's design, the degree of load sharing between fingers can be determined by selecting between two transmission stiffnesses based on actuation direction [20]. The geometry of the hands is constrained by 


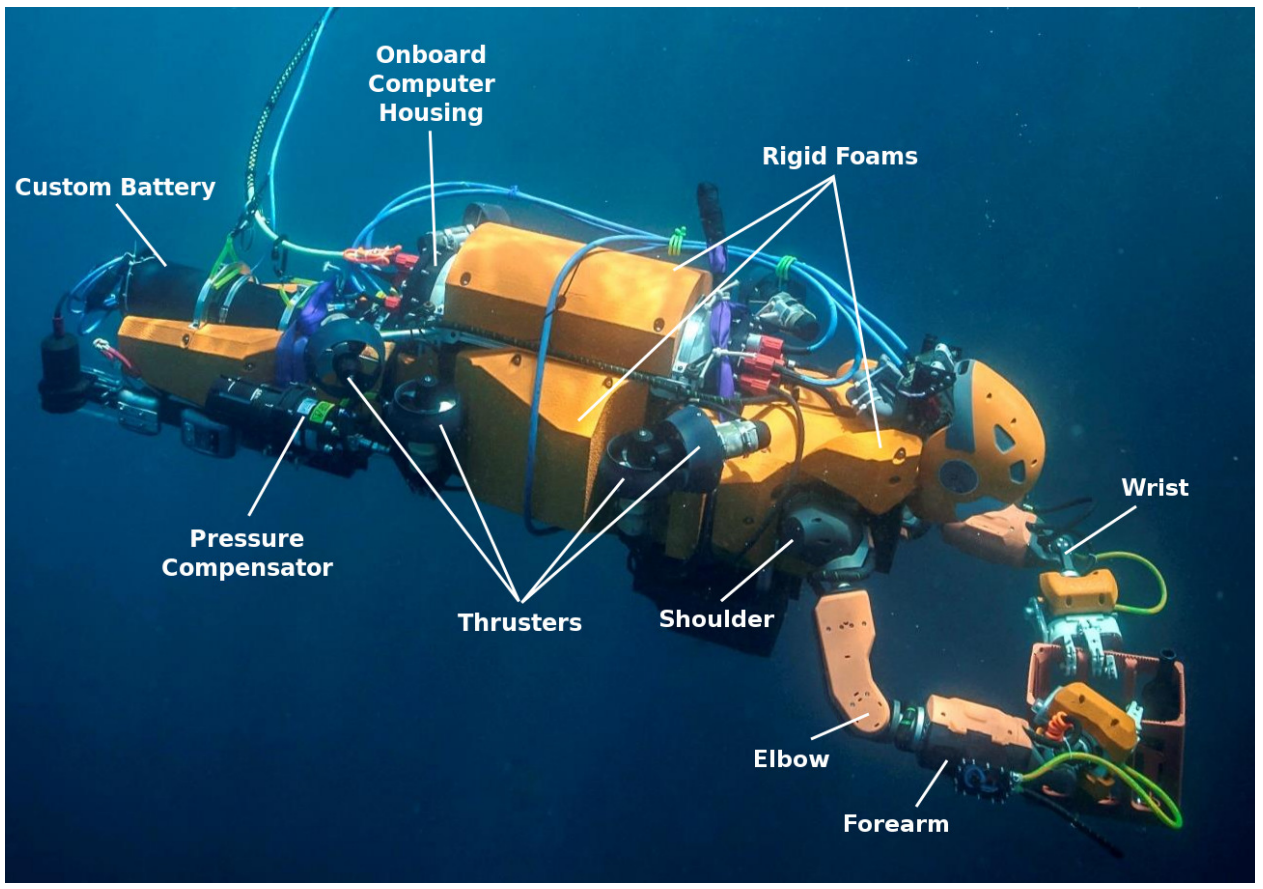

Fig. 4. Ocean One delivering a plastic crate. The body employs 8 thrusters, 4 on each side. Floatation foams create a neutrally buoyant platform. Spring-loaded oil-filled pressure compensators ensure that if a small leak occurs, the robot compartments do not take on water.
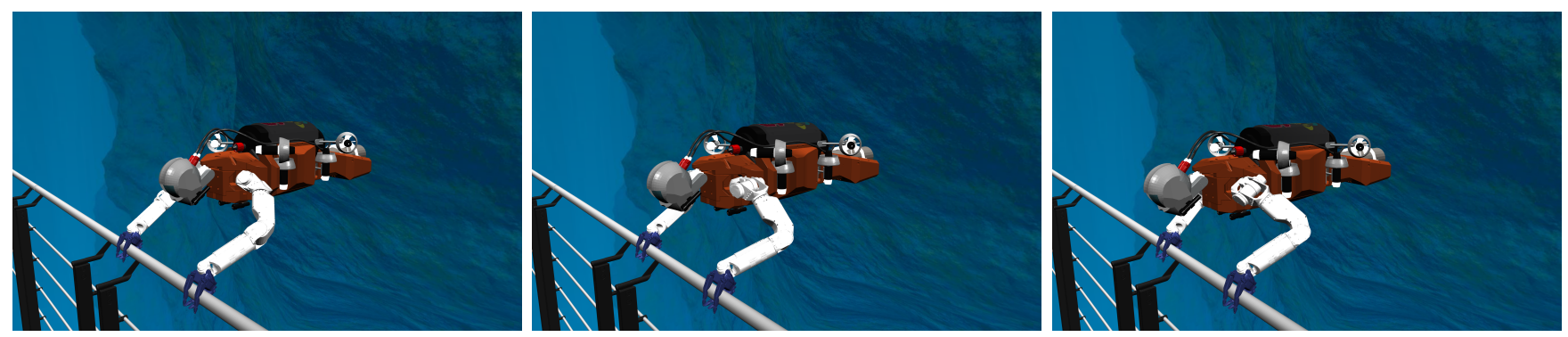

Fig. 5. During docking, the hands come to a complete halt while the arms continue to bend to dissipate the body inertial forces.

the need to achieve a variety of common grasps, including wrapping on heavy frames or tools and more delicate pinching [5], [7]. In particular, a wide finger base (Fig. 7 right) helps to stabilize long tools but, closing with straight fingers, the hand defaults to a parallel pinch of thin or small objects. Conversely, if the proximal phalanges make contact with the sides of a large object, the fingertips will move past each other to form an interlaced wrap grasp (Fig. 7 left).

The overall robot with twenty-four DOFs and twenty-six actuators is under $200 \mathrm{~kg}$ and is human-sized.

\section{CONTROL ARCHITECTURE}

Ocean One's on-board control architecture brings perception and action together in a multi-level structure as illustrated in Fig. 8, with increasing task abstraction at upper levels. The whole-body control level operates orders of magnitude faster than the elastic-planning and task-primitives level. Based on the operational space formulation of the robot's dynamics, the whole-body controller realizes motion and multi-contact task objectives while handling constraints, avoiding collisions, and coordinating the body-arms motions using the macro-mini high-bandwidth control strategy. This real-time controller operates with sensory feedback at $1 \mathrm{kHz}$, which allows for highly responsive interactions with the environment. Interaction forces at the hands are sensed through a 6-DOF force/torque sensor mounted at each wrist. Joint positions are measured using optical encoders, while the position and orientation of the body is estimated using an inertial measurement unit (IMU), a depth sensor and a Doppler velocity log (DVL).

The whole-body controller relies on task objectives generated through the elastic planning and task-primitive module. Elastic planning allows for real-time path modification in response to unforeseen changes in the environment [4]. The task primitive 

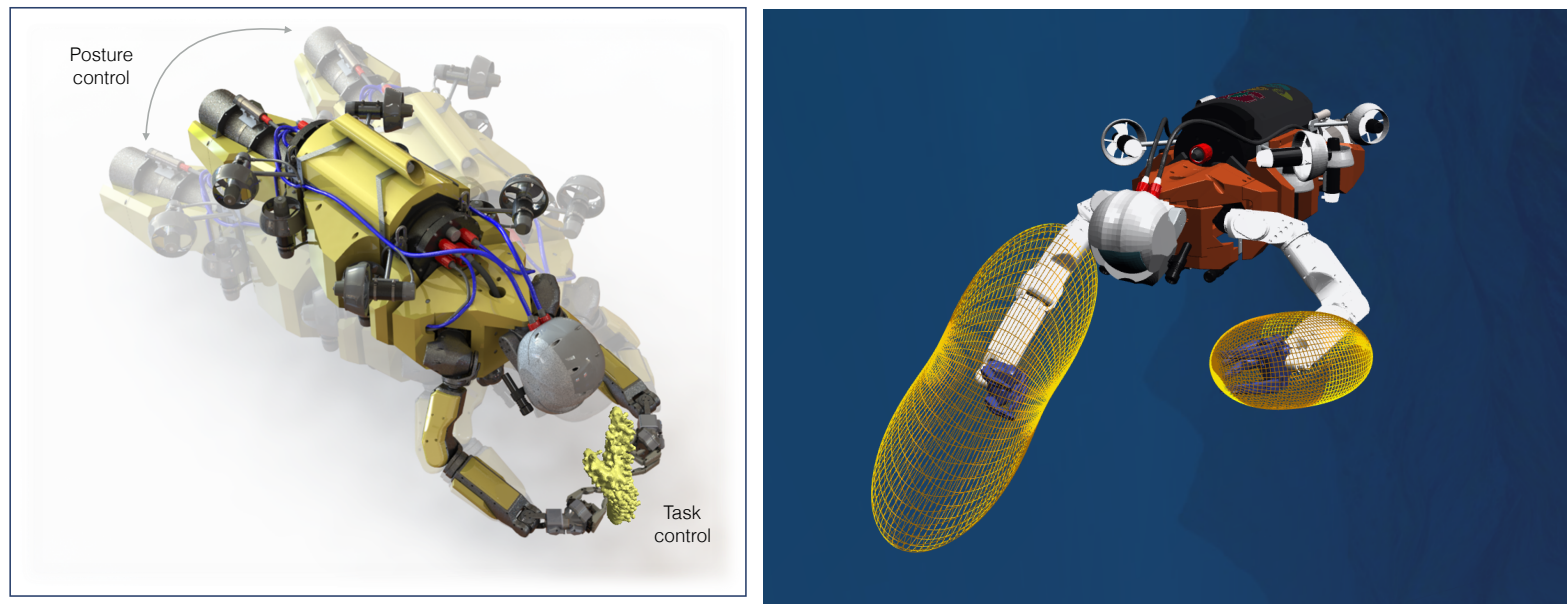

Fig. 6. Effect of design considerations on task dynamics. Left: Macro-mini dynamic co-ordination allows the task point to be stabilized in spite of large disturbances forces applied to the robot's body. The workspace and relative placement of the arms are optimized to maximize the range of arm motion over which the faster dynamics of the arms can be used to accommodate the slower dynamics of the body. Right: The yellow belted ellipsoids indicate the relative effective inertia at the each hand in different directions, and depends largely on the kinematics and configuration of the each arm.
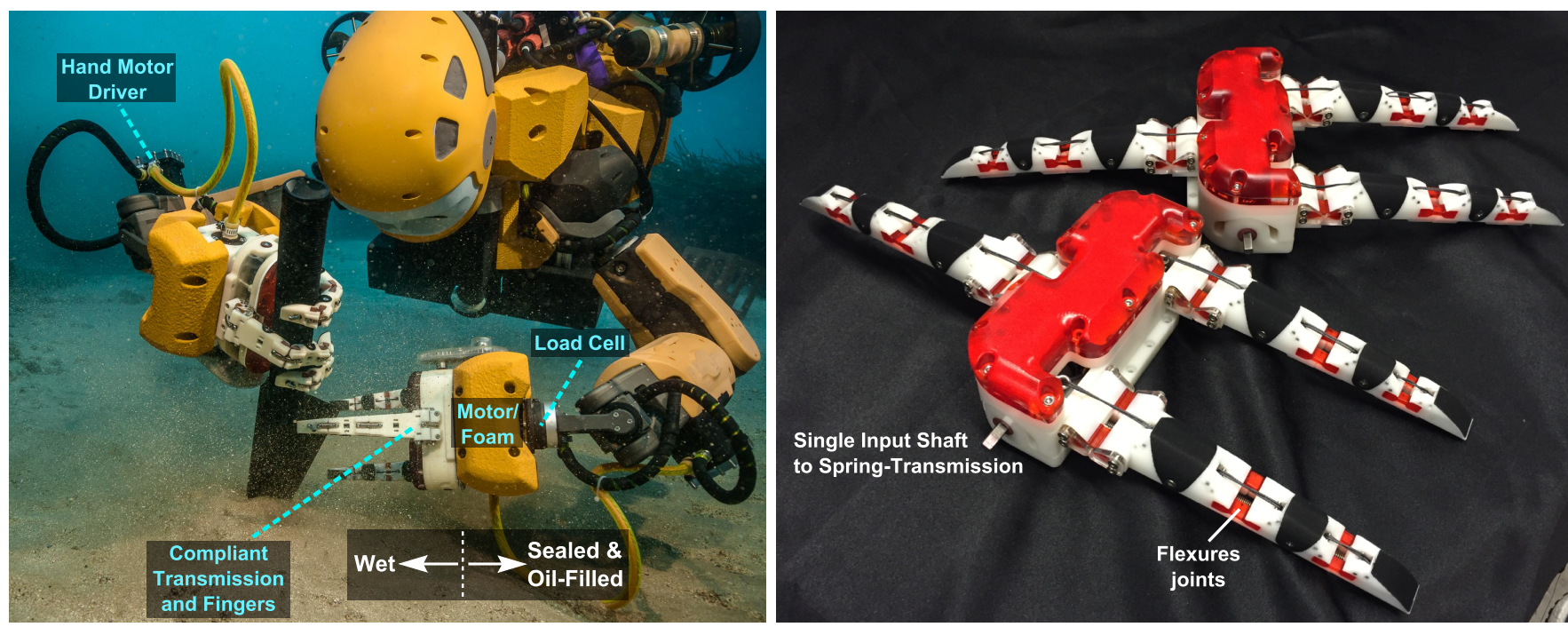

Fig. 7. Three-fingered compliant tendon-driven hands of Ocean One can pinch with straight fingers and perform interlaced wrap grasps. A back-drivable transmission allows the operator to choose between low and high transmission stiffness, depending on the task. Interaction forces are measured by a six-axis load cell at the wrist.

module provides an abstraction of the robot's manipulation skills in the form of a library of basic actions. The module relies on local and global models of the robot and its environment, which are maintained using the various on-board sensors and cameras.

The architecture is designed to allow the human user to interact with the robot and intervene at any level of task abstraction. At one end, the haptic devices directly output interaction forces to the whole-body controller. The robot mimics the motion of the pilot's hands at its own, while its senses are shared with the pilot - he sees what the robot sees and feels what the robot feels. At the other end, the robot autonomously executes a plan specified by the human operator through the elastic planning and task primitive module. The interface is designed to be as transparent as possible, in spite of communication delays resulting from remote operation. High fidelity haptic feedback is provided to the user using two Sigma.7 devices [11], while the user is visually immersed in the scene through a pair of binocular-stereo feeds presented on a passive 3D display (Fig. 9). A wide-angle body-mounted camera provides an additional view of the workspace.

\section{System VALidATION AND TESTING}

Preparation for the Lune mission was staged in two phases - simulation and in-pool, with simulation playing a critical role throughout Ocean One's overall design and development. Simulation and Active Interfaces (SAI) is our real-time interactive 


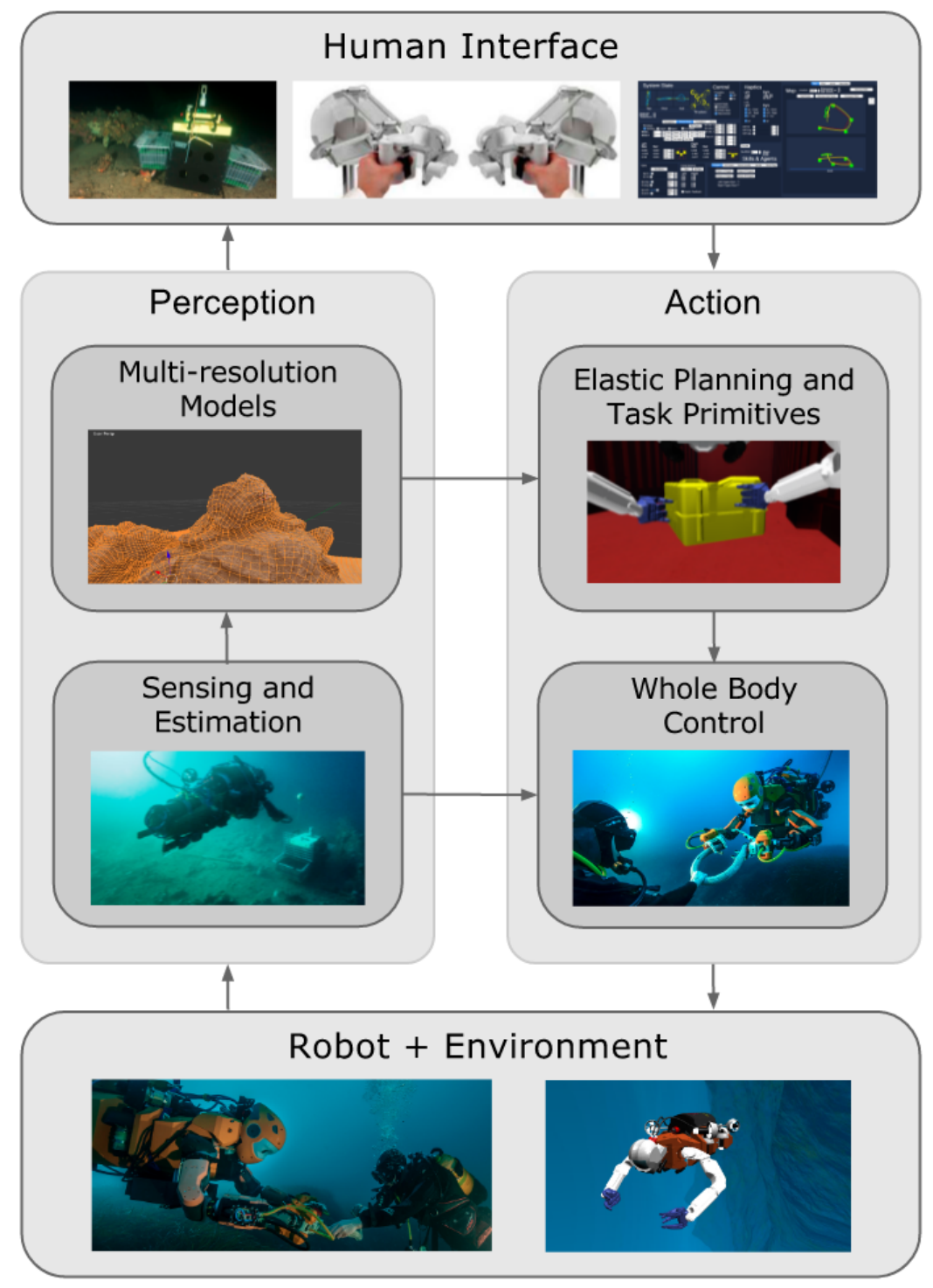

Fig. 8. Ocean One's control architecture combines perception and action in a hierarchical structure where task abstraction increases at upper levels. The user is able to interact with the robot and intervene at any level of task abstraction.

simulation environment for articulated body systems [14]. Critically, it enabled parallel development and testing of Ocean One's software from concept to fabrication. The simulation allows the pilot to guide the robot hands, while receiving haptic feedback from the virtual environment. The robot's body is autonomously coordinated, following the hands while avoiding obstacles. This feature was crucial to train pilots and to practice missions in their entirety.

With Ocean One designed to be operated only underwater, the Aquatic Center at Stanford University offers an ideal environment for testing hardware and system components (Fig. 10). The placement of foam parts and dead weights were adjusted for neutral buoyancy and balance. The robot was then controlled in the pool for various tasks using the haptic-visual interface, allowing to tune the control parameters. The UCC was designed to provide a display of the robot state and various sensors, select various control modalities and parameters, create motion plans, and record diagnostic data (Fig. 11). 


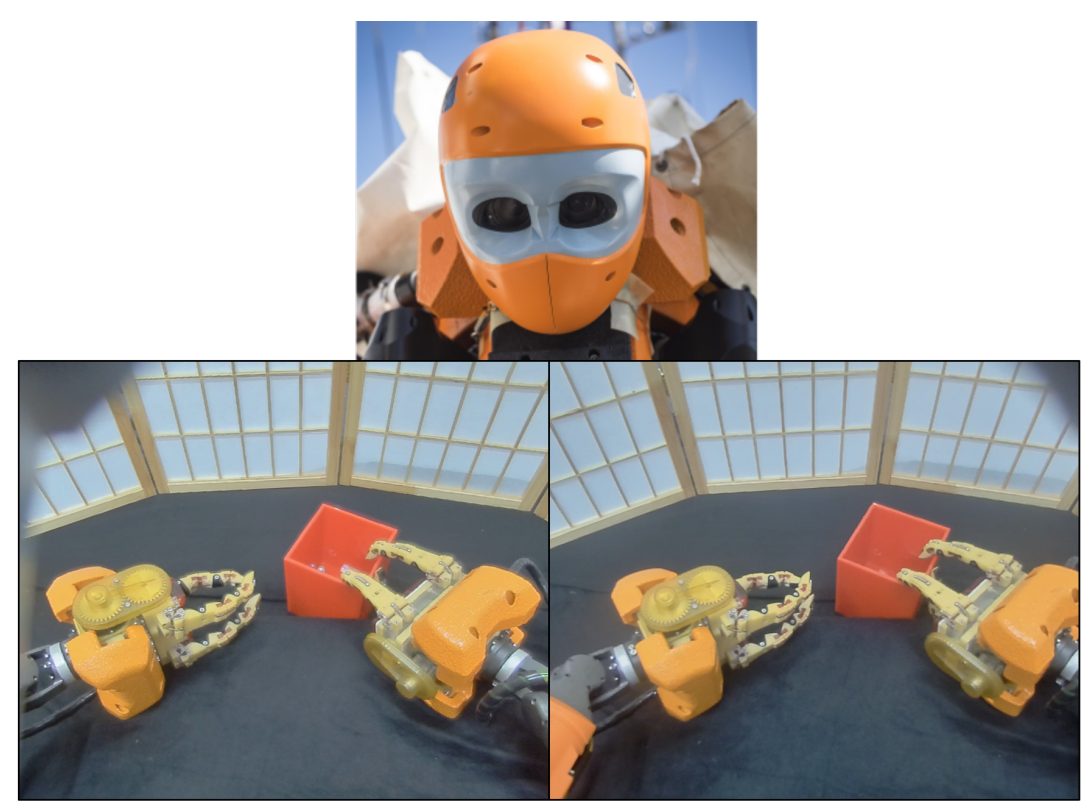

Fig. 9. Binocular-stereo cameras positioned at the robot's eyes stream to a passive 3D screen that immerses the operator in the task scene. A body-mounted camera (not shown) provides a wider alternative view.
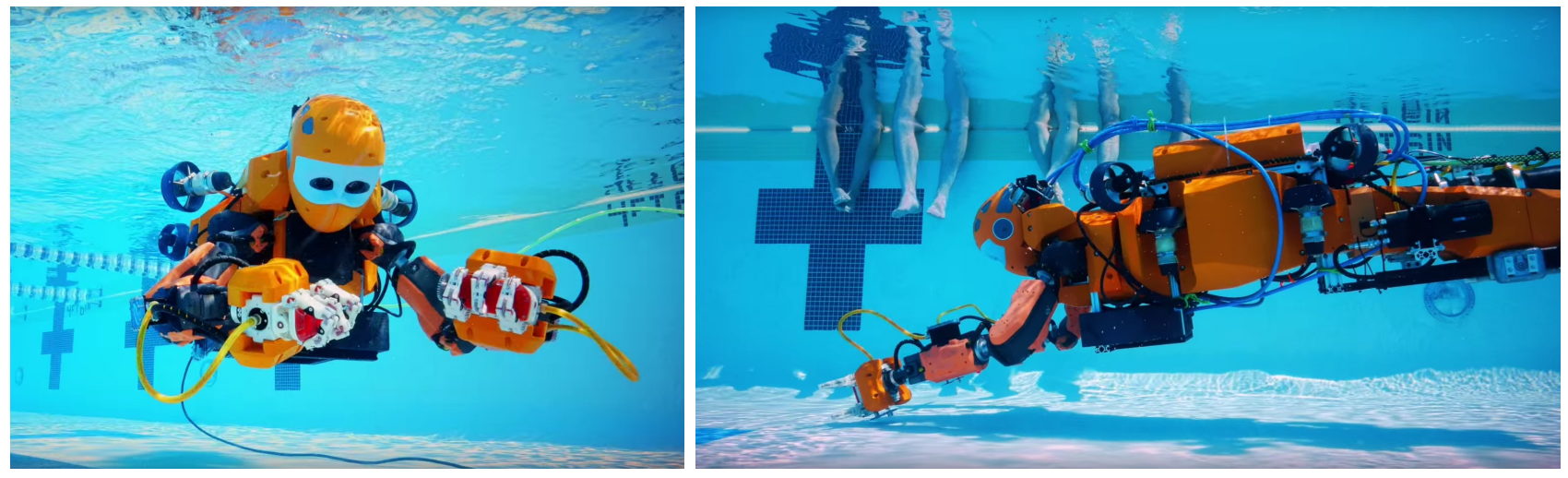

Fig. 10. Ocean One swimming at the Aquatic Center at Stanford University. This pool environment was used to adjust buoyancy and perform various validation tasks. 

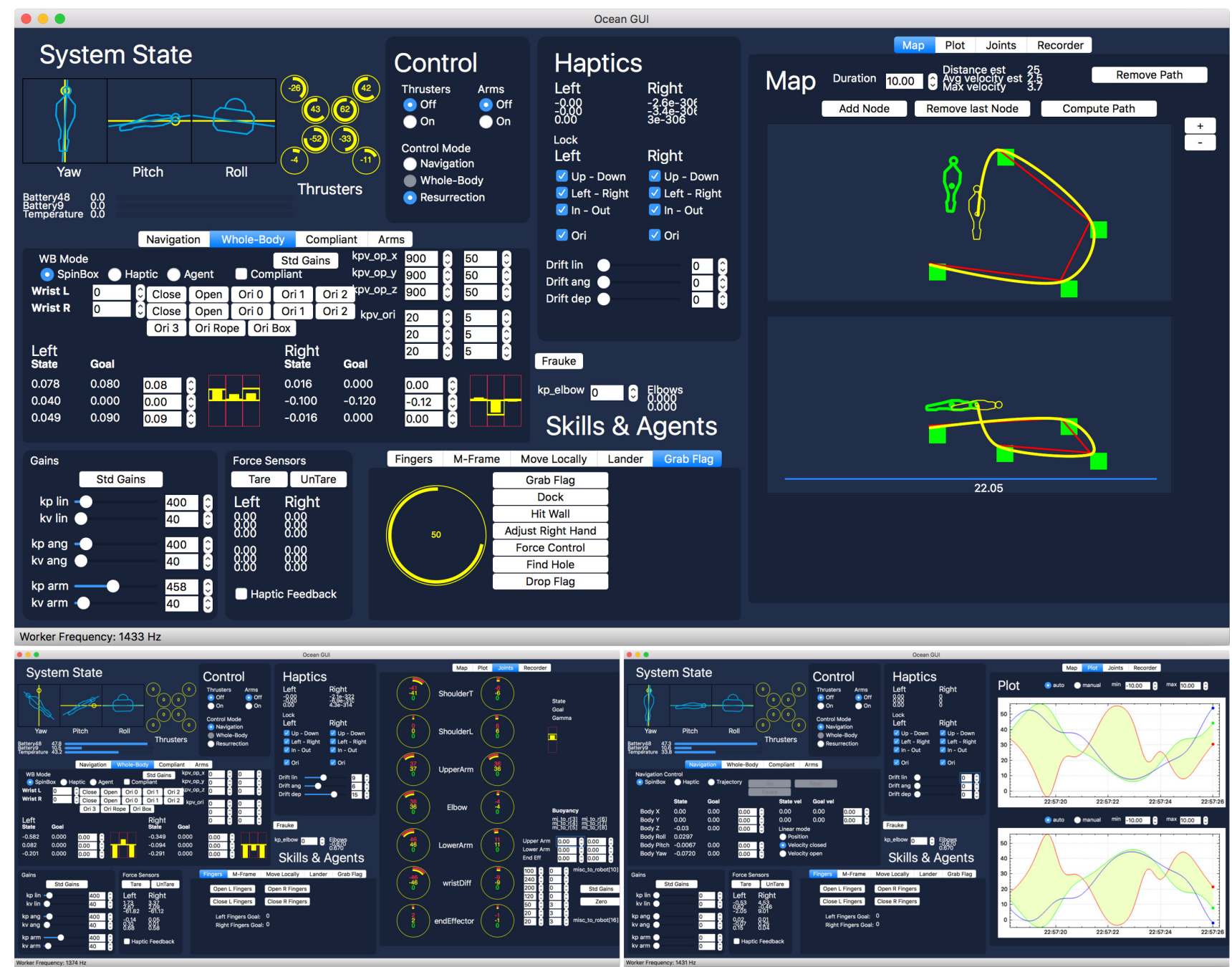

Fig. 11. The graphical User Command Center allows the user to monitor system state, select control modalities and plan manipulation tasks. Top: System state display - roll-pitch-yaw, depth, estimated linear planar position, thruster forces. Radio buttons for control mode selection. Push buttons and spin boxes for control parameter tuning. Interactive map that allows the operator to set trajectory control points (green squares), trajectory generation (yellow path), robot-state (green outline), robot-goal (yellow outline). Bottom-Left: Circular dial widgets displaying joint-space control state (red), goal (yellow), torque (green). Bottom-Right: Functionality for live plotting and recording for offline analysis. 


\section{EXPEDITION TO THE LUNE}

On April 6, 2016, Ocean One was flown from Stanford to Marseilles, France, for its maiden expedition to the Lune (French for 'moon'). The Lune was a two-decked fifty-four gun vessel at the cutting edge of the French Royal Navy in the first half of the seventeenth century. She took part in most of the naval battles of the first part of Louis XIV's reign and was memorialized in a painting by Puget (Fig. 12). Being supplanted by larger and more-heavily armed vessels and left in disuse, she was pressed into a relief mission to the coast of what is now Algeria. On return, she was denied entry to the port of Toulon over concerns for the plague and, in a damaged state, sank with nearly eight hundred aboard. Forgotten for over 300 years, she was discovered in May 1993 by Nautile, a submarine of the French Oceanographic Institute. The Lune offers a variety of artifacts including her artillery, equipment, and the personal belongings of crew and passengers, and is considered one of the greatest repositories of seventeenth century maritime, military, social and material history anywhere. Being at "only" 91 meters, she allows access for a robotic test dive while offering a preview of working conditions in much deeper water.

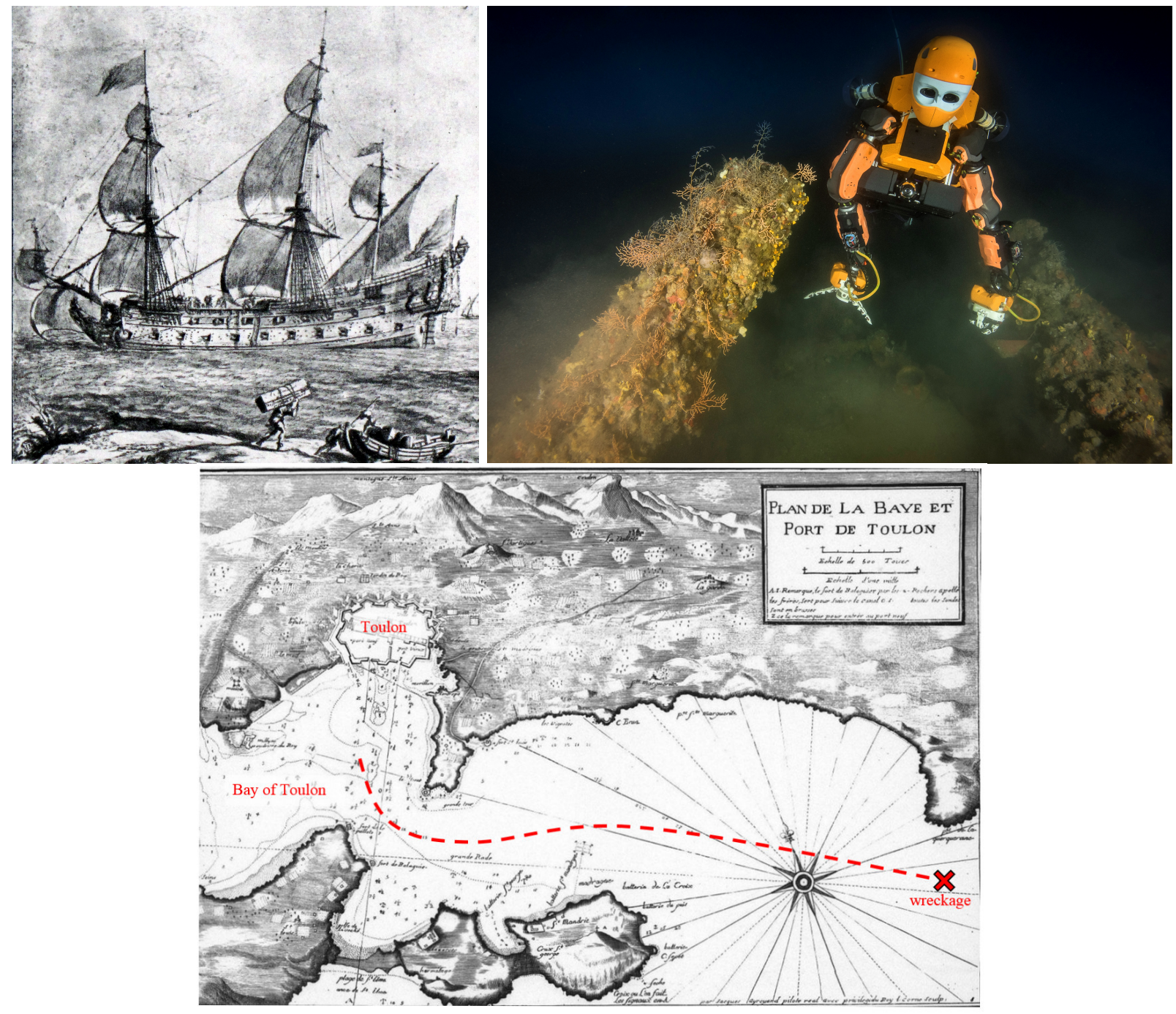

Fig. 12. La Lune, by Pierre Puget, 1654 (Louvre Museum, Paris, Graphical Arts Department INV 32594), is the only known representation of the ship before it sank in 1664 (left). Now, the surface of the wreckage is dominated by cannons, two of which Ocean One swims between (right). The Lune's course and resting site (below).

\section{André Malraux, the Host Ship}

There are as many as two hundred thousand archaeological sites lying off the Mediterranean and Atlantic coasts of France, and an estimated three million worldwide; the majority lie at depths inaccessible to human divers. To explore and protect these relics, DRASSM commissioned the André Malraux, a ship with state-of-the-art equipment for underwater archaeology including a remote-controlled robotic vehicle. Begun with the goal of providing remote exploration and maintenance capabilities to scientists above water, several on-site assessment and interaction capabilities were developed including 3D visualizations 
from mapping. Fig. 13 shows the operation room with the haptic-visual interface for Ocean One, together with the control interfaces for other supporting equipment.
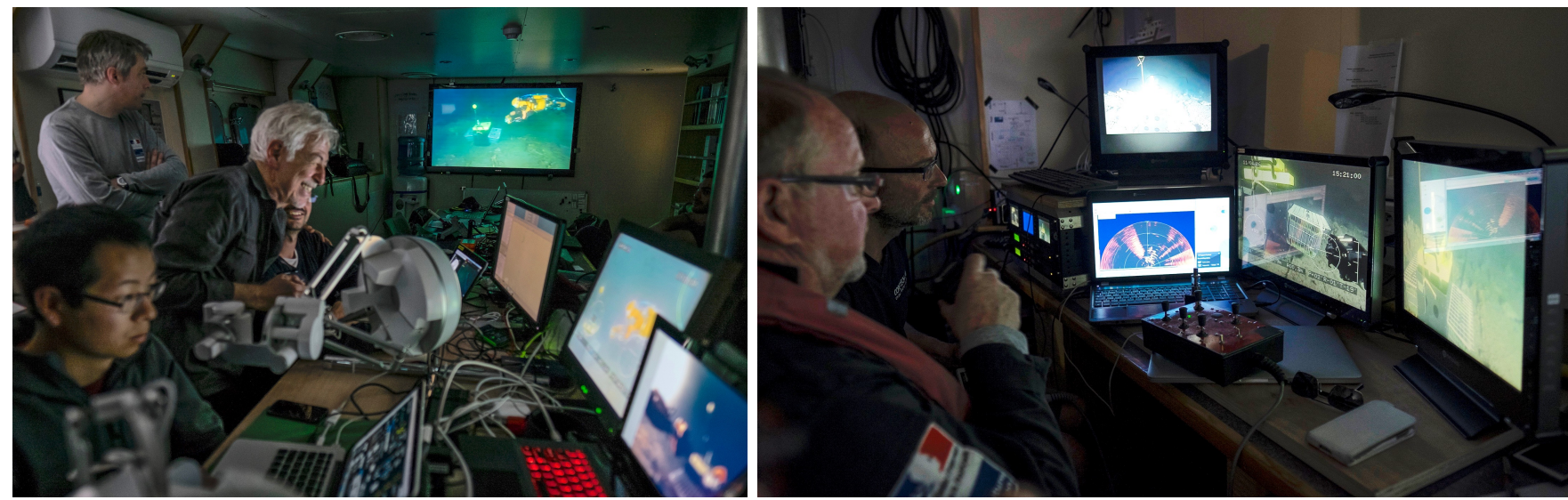

Fig. 13. The control room on-board André Malraux for this mission included the haptic-visual interface for Ocean One as well as views from other vehicles accompanying the robot during the mission.

\section{Field Support}

Once underwater, Ocean One was accompanied by three support ROVs: The mid-size working-class Perseo - equipped with a 5kW LED illumination system, a Sony HD professional video camera, and a Nikon D810 for stills; the LIRMM-designed research platform Leonard on a 3-meter tether above Ocean One and a downward looking camera for tracking; and the Achille for additional photography. In the control room, ROVs' operators coordinated to maintain separation and to compensate for tether tensions and currents.

\section{Mission at $15 \mathrm{~m}$ depth}

On April 11, Ocean One was lowered into the Mediterranean off the coast of Marseilles for its first operation at depth. The floor there being just $15 \mathrm{~m}$, with sand and sea life (Fig. 14), this enabled testing the system under field conditions with divers. The robot manipulated a piece of rope and a plastic crate with both hands; it also grasped and used a trowel. Divers interacted with the robot to test its cooperative manipulation capabilities. Interestingly, Ocean One and divers could communicate through hand gestures, as human divers often do.

\section{The Dive to the Lune}

The day after the successful $15 \mathrm{~m}$ mission, the André Malraux took Ocean One to the site of the Lune, six nautical miles off the coast of Toulon, where the robot made its way down $91 \mathrm{~m}$ to the ship. As the robot approached an area of the Lune located between two cannons, strong currents pushed it under one of them, where its left elbow and forearm became stuck. Navigating with thrusters alone failed to free Ocean One but, fortunately, through haptic interaction, it was able to use its arms to push itself free. Such maneuvering highlights the role of embodiment of the human form, intuition and haptic connection with the robot. After overcoming this close call, Ocean One was retrieved to the boat for inspection.

On April 15, Ocean One returned to the Lune to acquire an artifact. After exploring the site, a particular vase was selected. Ocean One grasped it by inserting one finger into its mouth and locking the others around it. Further securing the vase with the other hand, the robot delivered it to a sampling box (Fig. 15) equipped with an acoustically controlled float release. From there, the vase was raised to the surface and retrieved. 

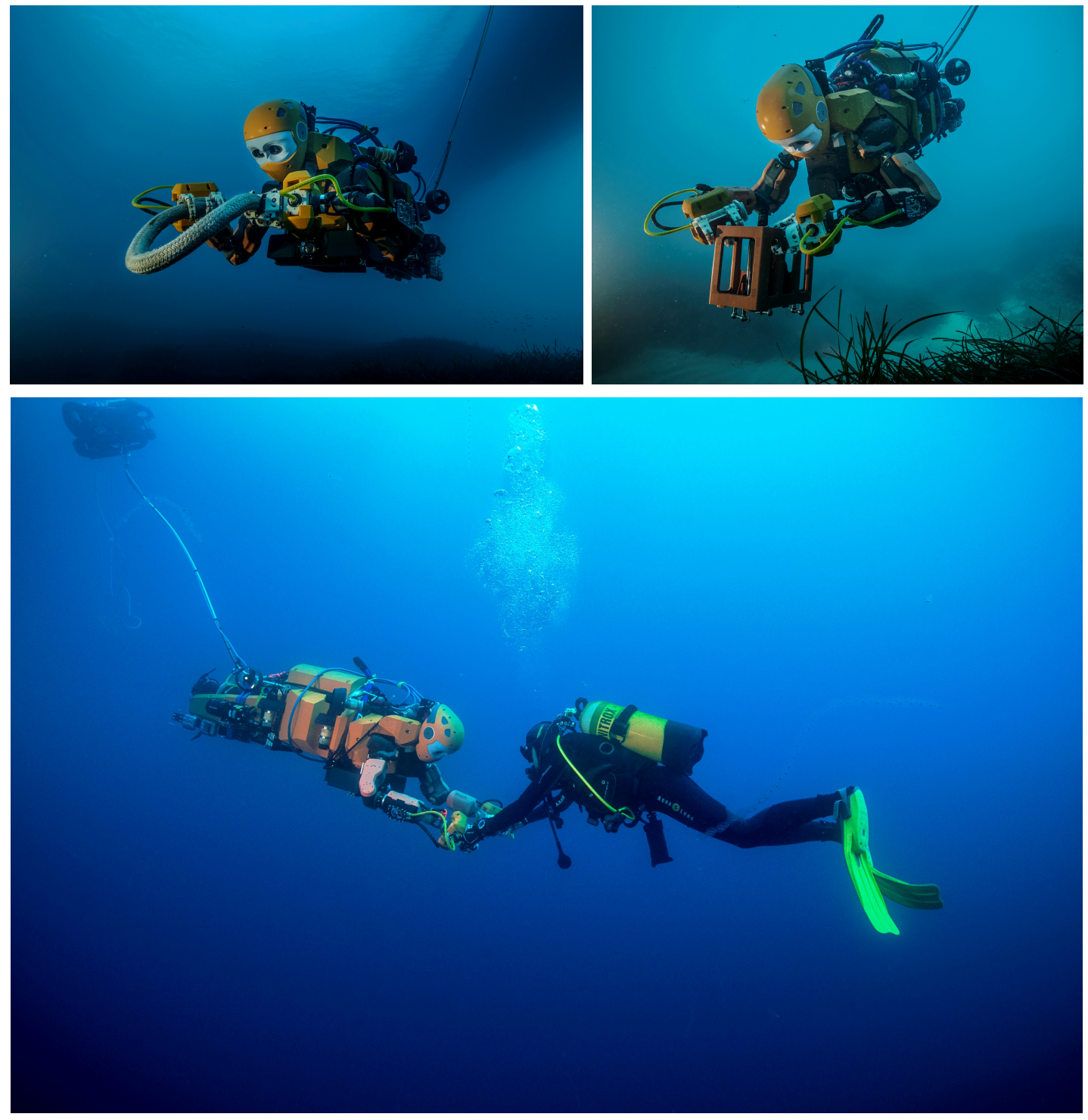

Fig. 14. Ocean One diving in the Mediterranean at $15 \mathrm{~m}$ : grasping objects (top) and interacting with the diver in a compliant and safe manner (bottom). 

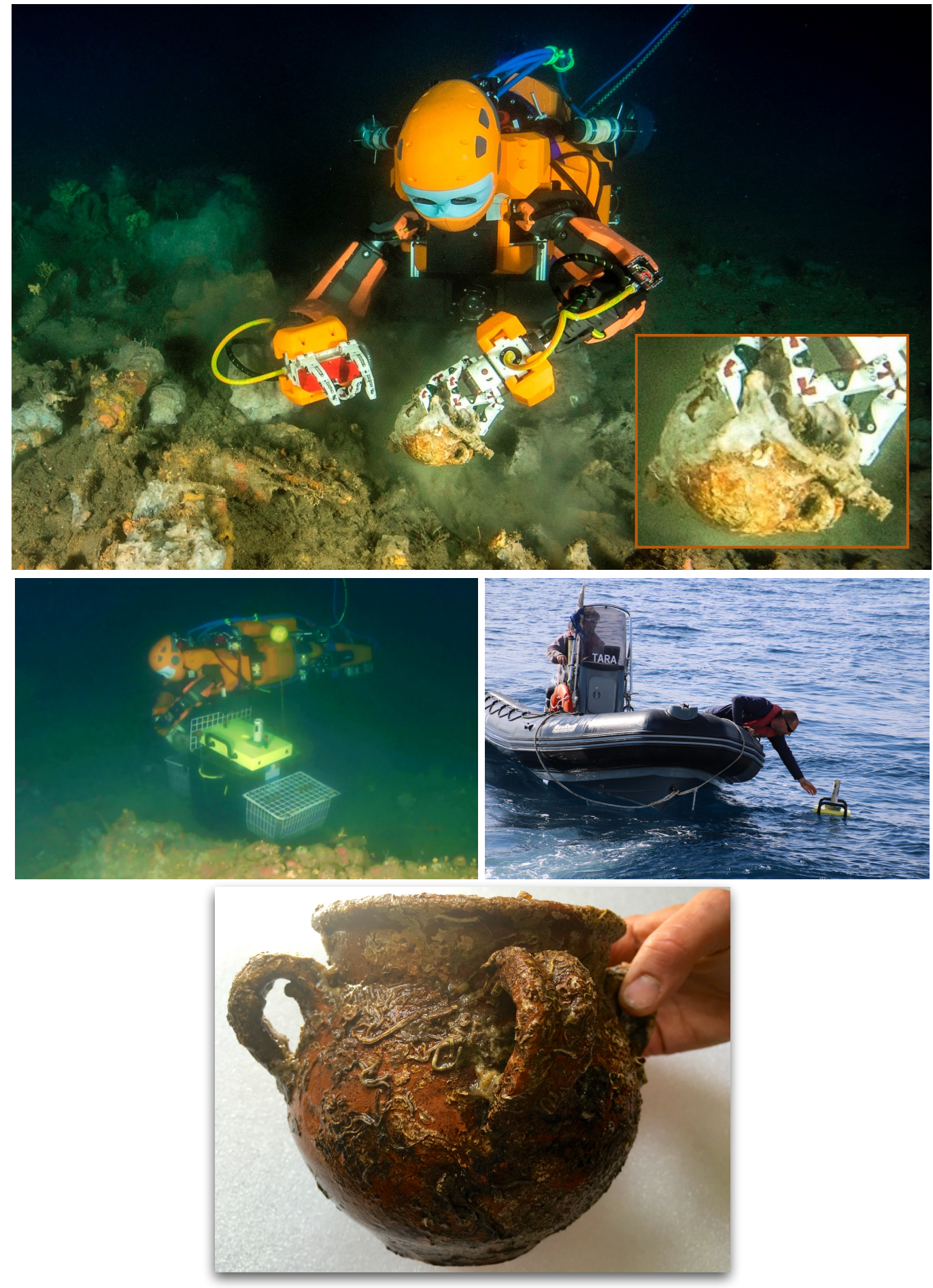

Fig. 15. Ocean One grasping a vase at the Lune site, detailed view of the grasping hand in the inset (top); artifact being placed in sample box and its retrieval at surface (middle); the recovered Catalan vase, seen with its acquired biofilm (bottom) 


\section{CONCLUSIONS}

The Ocean One expedition to the Lune demonstrated synergistic collaboration between robot and human, providing means for accomplishing challenging manipulation tasks in inhospitable environments. The key ingredients to its success were: $(i)$ the whole-body control and interaction skills of the robot balanced with expertise of the human, and (ii) the haptic-visual interface bringing a heightened sense of presence. Tasks such as coral reef monitoring, underwater pipeline maintenance, and mining demand these capabilities, and are planned for Ocean One.

More broadly, we believe Ocean One's accomplishments in the Mediterranean mark the beginning of a new level of manmachine cooperation. In the way that technological innovations of the past have impacted society - such as steam power, internal combustion engines, electro-mechanical systems, and digital computing - this ability to distance humans physically from dangerous and unreachable work spaces while connecting their skills, intuition and experience to the task may fundamentally alter remote work. We foresee that robotic avatars will search for and acquire materials in hazardous and inhospitable settings, maintain support equipment at remote sites, build infrastructure for monitoring the environment, and perform disaster prevention and recovery operations - be it deep in oceans and mines, at mountain tops, or in space.

\section{ACKNOWLEDGMENTS}

We would like to thank all the supporting partners and the students who have contributed so much to the development of Ocean One. The assistance of Harlyn Baker and Samir Menon in preparation of this manuscript is also gratefully acknowledged.

\section{Photo Credits}

$[1,4,7,12$ upper right] Frederic Osada and Teddy Seguin, DRASSM/Stanford University

[12 lower] Plan de la Baye et Port de Toulon (Jacques Ayrouard BNF. Cartes et Plans. GE DD-2987 (1404))

\section{REFERENCES}

[1] C. Amante and B.W. Eakins. Etopo1 1 arc-minute global relief model: Procedures, data sources and analysis. http://dx.doi.org/10.7289/V5C8276M, 2009.

[2] AUVAC, 86 Old Concord Turnpike, Lee, New Hampshire 03861 USA. Sauvim. http://auvac.org/configurations/view/754 (accessed: Dec. 14, 2013). Internet.

[3] L. Birglen, T. Laliberté, and C. Gosselin. Underactuated Robotic Hands, volume 40 of Springer Tracts in Advanced Robotics, chapter 1, Introduction, pages 1-5. Springer, Berlin, Heidelberg, Germany, first edition, 2008.

[4] O. Brock and O. Khatib. Elastic strips: A framework for motion generation in human environments. The International Journal of Robotics Research, 21(12):1031-1052, December 2002.

[5] I.M. Bullock, J.Z. Zheng, S. De La Rosa, C. Guertler, and A.M. Dollar. Grasp frequency and usage in daily household and machine shop tasks. IEEE Transactions on Haptics, 6(3):296 - 308, February 2013.

[6] M. C. Carrozza, C. Suppo, F. Sebastiani, B. Massa, F. Vecchi, R. Lazzarini, M. R. Cutkosky, and P. Dario. The SPRING hand: Development of a self-adaptive prosthesis for restoring natural grasping. Autonomous Robots, 16(2):125-141, March 2004.

[7] M. Cutkosky. On grasp choice, grasp models, and the design of hands for manufacturing tasks. IEEE Transactions on Haptics, 5(3):269 - 279, June 1989.

[8] Force Dimension. Homepage. http://www.forcedimension.com (accessed: Feb. 24, 2015). Internet, 2015.

[9] A. Dollar and R. D. Howe. The highly adaptive SDM hand: Design and performance evaluation. The International Journal of Robotics Research, 29(5):585-597, April 2010.

[10] ECA Group. Homepage. http://www.ecagroup.com/en/solutions/arm-5e-micro (accessed: Sep. 18, 2016). Internet, 2016.

[11] Force Dimension, Route de Saint-Cergue 295, CH-1260 Nyon, Switzerland. Homepage. http://www.forcedimension.com (accessed: Nov. 26, 2010). Internet, 2010.

[12] Kevin C. Galloway, Kaitlyn P. Becker, Brennan Phillips, Jordan Kirby, Stephen Licht, Dan Tchernov, Robert J. Wood, and David F. Gruber. Soft Robotic Grippers for Biological Sampling on Deep Reefs. Soft Robotics, 00(00):soro.2015.0019, 2016.

[13] O. Khatib. Inertial properties in robotics manipulation: An object-level framework. The International Journal of Robotics Research, 14(1):19-36, feb 1995.

[14] O. Khatib, L. Sentis, J. Park, and J. Warren. Whole-body dynamic behavior and control of human-like robots. International Journal of Humanoid Robotics, 1(1):29-43, 2004.

[15] D.M. Lane, J.B.C. Davies, G. Robinson, D.J. O’Brien, J. Sneddon, E. Seaton, and A. Elfstrom. The AMADEUS dextrous subsea hand: design, modeling, and sensor processing. IEEE Journal of Oceanic Engineering, 24(1):96-111, 1999.

[16] Parker Hannifin Corp. Homepage. http://http://www.parker.com/ (accessed: Feb. 3, 2012). Internet, 2012.

[17] G. Pratt and M. Williamson. Series elastic actuators. In Proc. of the IEEE/RSJ International Conference on Intelligent Robots and Systems, volume 1, pages 399-406, Pittsburgh, PA, USA, August 1995.

[18] P.J. Sanz, M. Prats, P. Ridao, D. Ribas, G. Oliver, and A. Ortiz. Recent progress in the rauvi project: A reconfigurable autonomous underwater vehicle for intervention. In ELMAR, 2010 PROCEEDINGS, pages 471-474, Sept 2010.

[19] Schilling Robotics, LLC, 260 Cousteau Place, Davis, CA 95618, USA. Homepage. http://www.schilling.com (accessed: Dec. 14, 2011). Internet, 2011.

[20] H. Stuart, S. Wang, O. Khatib, and M.R. Cutkosky. The Ocean One Hands : An Adaptive Design for Robust Marine Manipulation. Manuscript submitted for publication.

[21] H.S. Stuart, S. Wang, B. Gardineer, D.L Christensen, D.M. Aukes, and M. Cutkosky. A compliant underactuated hand with suction flow for underwater mobile manipulation. In Proc. of the IEEE International Conference on Robotics and Automation, pages 6691 - 6697, Hong Kong, May 2014.

[22] M. Zinn, B. Roth, O. Khatib, and J.K. Salisbury. A new actuation approach for human friendly robot design. The International Journal of Robotics Research, 23(4):379-398, 2004. 